\title{
A Study on Axial Bearings Lubrication Characteristics in High-speed Planetary Transmissions of Vehicles
}

\author{
Hong-wei Wang ", Kai Huang, and Yuan Su \\ College of Mechanical and Equipment Engineering, \\ Hebei University of Engineering, Handan, 056038, China
}

\section{Keywords: Planetary Transmissions, Axial Bearings, Lubricating Characteristics}

\begin{abstract}
To reduce the wear of thrust washer within straight planetary gear set, hydrodynamic lubrication design was studied by way of machining grooves in the radial direction into sides of thrust washer. Such grooves can result in a hydrodynamic pressure to separate the contact surfaces and reduce the wear accordingly. Based on mass boundary conditions, the Reynolds equation was discretized using control volume method. The results show that, to increase the axial clearance and groove depth, to decrease groove width ratio and groove number can decrease the friction between planetary gear transverse and axial bearing surface. The fluid flow increased when groove depth and groove numbers increased. The bearings have the maximum load capacity when the groove number is 24 and the groove width ratio is 0.5 . In engineering applications, make sure the groove depth is $10 \sim 40 \mu \mathrm{m}$, and recommend assembling clearance between planet wheel and thrust washer is $5 \mu \mathrm{m}$.
\end{abstract}

\section{Introduction}

High performance, high power density, high reliability and long life are the development trend of vehicle transmission technology, and improved the speed is an effective way to improve power density [1]. In the automatic transmission of the vehicle the helical planetary row is used for transmission. The axial bearing between the planetary gear carrier surface and the planet carrier is installed, usually the clearance of surfaces between the two don't exceed $1 \mathrm{~mm}$ [2]. In consideration of the bilateral rotation of the gear shaft to the bearing and the strict clearance requirements, the components are selected by a radial straight groove thrust washer [3]. The thrust washer uses the dynamic pressure created by the fluid to achieve a non-contact state with the high speed planetary wheel, instead of the previous contact friction. The friction between the end faces is reduced to extend the service life of the transmission. The foreign research of non-contact axial support earlier research literature on [4-5] bevel gear planetary disc row axial supporting by theory and experiment, proved that the radial slot can bear axial hydrodynamic pressure generated between the load, improve the axial bearing capacity of fluid between the Ref. [6] studied different groove parameters and end the influence of bearing capacity. It is pointed out that under different conditions, the existence of the optimal value of groove parameters. The domestic literature for [7-8] analysis of compound planetary gear axial supporting roller, wear and axial force effect, analysis focused on contact friction movement; for non mechanical contact face main aspects of research on the mechanical seal [9-11]. The application of pressure to support the vehicle high speed planetary axle using fluid ladder trough, Ref. [12] on the thrust washer fluid inlet and outlet pressure difference lubrication mechanism analysis of the impact of groove parameters on the flow rate, the temperature rise of the washer, the paper points out that the radial line through groove can increase the axial flow to take away the heat generated by friction and according to the parameters optimization of actual conditions to improve the axial bearing capacity. According to the planetary gear speed generated by the friction heat causes the oil film temperature and reduce the bearing capacity of the planet wheel axle to Inter, the thrust washer fluid inlet and outlet pressure difference increasing flow of thrust washer model of fluid lubrication mechanism, parameter analysis and optimization, to reduce the vehicle speed to the planet wheel axle the abrasion and improve the face of oil film opening force of theoretical analysis and discussion. 


\section{Geometric Model}

A structure of axial parallel bearing end surface with opened micron trench, which is divided into groove zones and station zones shown in Fig. 1.

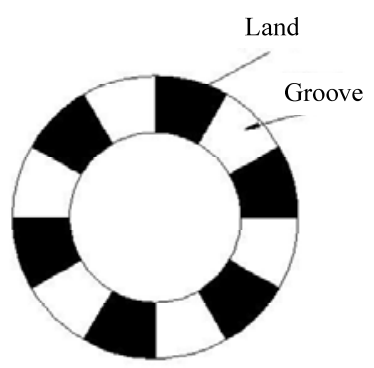

Figure 1. Axial bearing with radial groove

A period in a straight line groove is defined to include a land area and an adjacent groove area because it has a periodicity. The geometric model for one period is shown in Fig. 2.

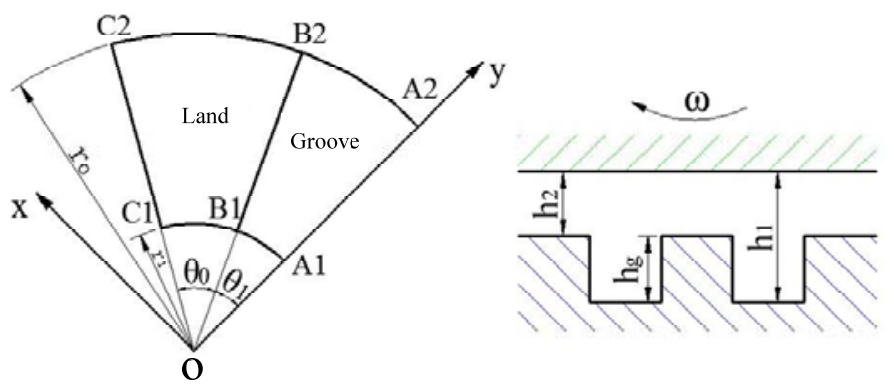

Figure 2. A periodic geometric model

Description of each physical quantity: ri and ro are axial bearing inner and outer radius, A1-A2, B1-B2 and C1-C2 are the boundary lines of a periodic slot, $\theta 0$ is the angle of the periodic groove, $\theta 1$ is the angle of the periodic table area, $\theta 1 /(\theta 0+\theta 1)$ is a slot width ratio expressed as $d, h 1$ is the thickness of oil film in groove area, $\mathrm{h} 2$ is the thickness of oil film in a platform.(The oil film thickness which is formed by the side of the planet gear and the thrust washer in the table area is also called clearance), hg is the depth of the groove.

Boundary conditions are divided into deterministic boundary condition and periodic boundary condition.

$$
\left\{\begin{array}{l}
\bar{r}=1, \bar{p}=\bar{p}_{\mathrm{i}} \\
\bar{r}=\bar{r}_{\mathrm{o}}, \bar{p}=1 \\
\left.\bar{p}\right|_{\theta=0}=\left.\bar{p}\right|_{\theta=2 / N_{\mathrm{g}}}
\end{array}\right.
$$

Where $\mathrm{N}$ is the groove number, and $\theta$ is the circumferential angle of axial bearing.

\section{Mathematical Model}

\section{Model Assumptions}

Axial support for the stationary state is assumed in this study, but the rotation speed of the planet wheel is kept constant. Also, the following assumptions were made in the derivation of the fluid momentum equation:(1) The fluid flow maintains a laminar flow state;(2) Lubricant viscosity is 
constant;(3) The sliding bearing surface is parallel to the planetary gear working surface and all surfaces are rigid;(4) Neglecting the influence of the fluid inertia force.

\section{Reynolds Equation}

In the stable condition, when the washer which processing with radial rectangular groove and a planetary wheel moves relatively, lubricant flow between them obey steady Reynolds equation[13], as shown in formula (2).

$$
\frac{\partial}{\partial x}\left(\frac{h^{3}}{\mu} \frac{\partial p}{\partial x}\right)+\frac{\partial}{\partial y}\left(\frac{h^{3}}{\mu} \frac{\partial p}{\partial y}\right)=6 U \frac{\partial h}{\partial x}
$$

Where $\mu$ is the dynamic viscosity of lubricating oil, $\mathrm{h}$ is film thickness and $\mathrm{U}$ is fluid velocity with the $\mathrm{X}$-axis positive.

The expression written in cylindrical coordinates is given

$$
\frac{1}{r} \frac{\partial}{\partial \theta}\left(\frac{h^{3}}{\mu} \frac{\partial p}{\partial \theta}\right)+\frac{\partial}{\partial r}\left(\frac{r h^{3}}{\mu} \frac{\partial p}{\partial r}\right)=6 r \omega \frac{\partial h}{\partial \theta}
$$

Where, a rotation angular velocity $\omega$ for wheel and axial bearing radius $r$ are utilized in Eq. (3).

In order to facilitate formula derivation, the vector form is given

$$
\nabla \cdot\left(-h^{3} \nabla p+6 \mu r \omega h \hat{\theta}\right)=0
$$

The non-dimensional of above equation is given

$$
\nabla \cdot\left(-\bar{h}^{3} \nabla \bar{p}+\gamma \bar{r} \bar{h} \hat{\theta}\right)=0
$$

The above non-dimensional parameter is defined as

$$
\bar{r}=\frac{r}{r_{\mathrm{i}}}, \bar{p}=\frac{p}{p_{\mathrm{o}}}, \quad \bar{h}=\frac{h}{h_{\mathrm{g}}}, \quad \gamma=\frac{6 \mu \omega r_{\mathrm{i}}^{2}}{p_{\mathrm{o}} h_{\mathrm{g}}^{2}}
$$

The vector field $\left(-\bar{h}^{3} \nabla \bar{p}+\gamma \bar{r} \bar{h} \hat{\theta}\right)$ divergence in Eq. (5) is zero, so the vector field is a passive field, it is shown that the integral is zero in a closed region, therefore:

$$
\oint_{\Gamma}\left\{-\bar{h}^{3} \nabla \bar{p}+\gamma \bar{r} \bar{h} \hat{\theta}\right\} \cdot n d \Gamma=0
$$

Where, $\Gamma$ is the control body boundary, $\mathrm{n}$ is the unit vector of normal direction outside the closed area, $\mathrm{d} \Gamma$ is the length of the boundary element.

The physical meaning of Eq. (7) is when the planetary wheel shaft is stable to the support, the net mass of the body is zero, the mass of the inflow control body and the mass vector of the outflow control body are zero.

Integral to Eq. (7), the non-dimensional flow through the boundary of the control body is given 


$$
\begin{gathered}
\bar{Q}_{r}=\int_{0}^{\theta_{0}}\left(-\bar{h}^{3} \frac{\partial \bar{p}}{\partial \bar{r}}\right) \bar{r} d \theta \\
\bar{Q}_{\theta}=\int_{1}^{\bar{r}_{o}}\left(-\bar{h}^{3} \frac{1}{\bar{r}} \frac{\partial \bar{p}}{\partial \bar{\theta}}+\gamma \bar{r} \bar{h} \hat{\theta}\right) d \bar{r}
\end{gathered}
$$

The half step grid discretization was used for the selection of control volume difference scheme. Along the radial direction is $r$, denoted by $\mathrm{i}$; Axial direction is $\theta$, denoted by $\mathrm{j}$, grid nodes are numbered sequentially. The solution domain is a two-dimensional mesh boundary $\Gamma$, the boundary of the region is divided into six element length, respectively is ABCDEF, the flow through the control boundary is shown in Fig. (3).

Radial straight through the inlet and outlet area net flow is zero, according to the principle of mass conservation, the non-dimensional form of the control flow is satisfied.

$$
\bar{Q}_{r}^{A}+\bar{Q}_{r}^{B}+\bar{Q}_{\theta}^{F}-\bar{Q}_{r}^{E}-\bar{Q}_{r}^{D}-\bar{Q}_{\theta}^{C}=0
$$

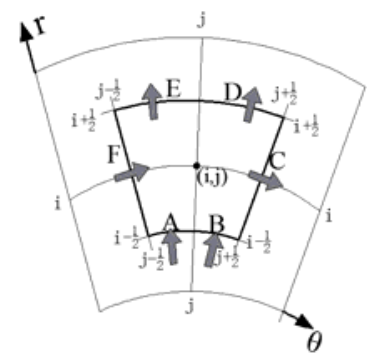

Figure 3. Schematic diagram of control volume

The control volume method is used in the discrete form of the equation, in order to improve the accuracy of calculation, The second-order central difference scheme is adopted for each numerical calculation, The discrete numerical expression for the finite control domain is derived

$$
\left\{\begin{array}{l}
\bar{Q}_{r}^{A}=-\bar{h}_{A}^{3} \frac{\bar{p}_{i, j}-\bar{p}_{i-1, j}}{\Delta \bar{r}} \frac{\bar{r}_{i-1, j}+\bar{r}_{i, j}}{2} \Delta \theta \\
\bar{Q}_{r}^{B}=-\bar{h}_{B}^{3} \frac{\bar{p}_{i, j}-\bar{p}_{i-1, j}}{\Delta \bar{r}} \frac{\bar{r}_{i-1, j}+\bar{r}_{i, j}}{2} \Delta \theta \\
\bar{Q}_{\theta}^{C}=\left(-\frac{\bar{h}_{C}^{3}}{\bar{r}_{i, j}} \frac{\bar{p}_{i, j+1}-\bar{p}_{i, j}}{\Delta \theta}+\gamma \bar{r}_{i, j} \bar{h}_{C}\right) \cdot \Delta \bar{r} \\
\bar{Q}_{r}^{D}=-\bar{h}_{D}^{3} \frac{\bar{p}_{i+1, j}-\bar{p}_{i, j}}{\Delta \bar{r}} \frac{\bar{r}_{i+1, j}+\bar{r}_{i, j}}{2} \Delta \theta \\
\bar{Q}_{r}^{E}=-\bar{h}_{E}^{3} \frac{\bar{p}_{i+1, j}-\bar{p}_{i, j}}{\Delta \bar{r}} \frac{\bar{r}_{i+1, j}+\bar{r}_{i, j}}{2} \Delta \theta \\
\bar{Q}_{\theta}^{F}=\left(-\bar{h}_{F}^{3} \frac{\bar{p}_{i, j}-\bar{p}_{i, j-1}}{\Delta \theta}+\gamma \bar{r}_{i, j} \bar{h}_{F}\right) \cdot \Delta \bar{r}
\end{array}\right.
$$

The discretization of the expression of the radial straight-line difference scheme based on the control volume method in each grid node constitutes a system of equations, the coefficients in the 
equation are the functions of the oil pressure and film thickness of each node, which can form (m-1) $\times(n-1)$ linear non-homogeneous systems. numerical iteration algorithm is used to solve the fluid pressure field. The Gauss-Seidel relaxation iteration is used to achieve good convergence in the paper, Convergence condition is given

$$
\varepsilon=\sum_{i=1}^{m+1} \sum_{j=1}^{n+1}\left|\frac{\bar{p}_{i, j}^{(k+1)}-\bar{p}_{i, j}^{(k)}}{\bar{p}_{i, j}^{(k+1)}}\right| \leq[\varepsilon]
$$

Take $[\varepsilon]=10-6$. The correction function of the non-dimensional pressure in each iteration is given

$$
\bar{p}_{i, j}^{(k+1)}=\bar{p}_{i, j}^{(k)}+\kappa\left(\tilde{p}_{i, j}^{(k+1)}-\bar{p}_{i, j}^{(k)}\right)
$$

In order to speed up the convergence rate, the relaxation factor $\kappa$ set as 1.8 , where the results of the last iteration are used for each iteration of the pressure.

The pressure field of the oil film is solved by numerical calculation, and then the influence of the groove type parameters on the lubrication characteristics is analyzed.

Non-Dimensional oil film bearing capacity

$$
\bar{W}_{z}=\int_{0}^{2 \pi} \int_{1}^{\bar{r}_{o}} \bar{p} \bar{r} d \bar{r} d \theta
$$

Non-dimensional friction torque

$$
\bar{T}_{f}=-\int_{0}^{2 \pi} \int_{1}^{\bar{r}_{o}} \frac{\partial \bar{h}}{\partial \theta} \bar{r} d \bar{r} d \theta-\frac{\gamma}{3} \int_{0}^{2 \pi} \int_{1}^{\bar{r}_{o}} \frac{\bar{r}}{\bar{h}} d \bar{r} d \theta
$$

Non-dimensional flow rate

$$
\bar{Q}_{r}=\left\{-\int_{0}^{2 \pi} \bar{h}^{3} \frac{\partial \bar{p}}{\partial \bar{r}} \bar{r} d \theta+\frac{\gamma}{20} \int_{0}^{2 \pi-2} \bar{h}^{3} d \theta\right\}_{\bar{r}_{o}}
$$

\section{Results and Discussion}

Axial bearing calculation using the specific parameters are as follows: rotational speed $n=6000$ $\mathrm{r} / \mathrm{min}$, groove depth $h_{\mathrm{g}}=3.2 \mu \mathrm{m}$, groove width ratio $K=0.5$, groove clearance $h_{2}=3.2 \mu \mathrm{m}$, groove number $N_{\mathrm{g}}=8$, the inner diameter $r_{\mathrm{i}}=17.5 \mathrm{~mm}$, outer diameter $r_{\mathrm{o}}=25 \mathrm{~mm}$, the inlet pressure $p_{\mathrm{i}}=1 \mathrm{Mpa}$, the inlet pressure $p_{0}=0.1 \mathrm{Mpa}$.

\section{Film Bearing Capacity Analysis}

The oil film bearing capacity of the surface is the sum of the thrust force acting on the side surface of the high speed planetary roller bearing (generated by hydrodynamic and static pressure). The effect of axial bearing parameters on the bearing capacity of the surface shows in Fig. 4. Influence of the groove width ratio on the bearing capacity of oil film is shown in Fig. 4a, it can be seen that the axial bearing capacity has maximum capacity when $\mathrm{K}=0.5$. The bearing capacity increases with the increase of groove width ratio when $\mathrm{K}<0.5$, however, it has the opposite result the bearing capacity when $\mathrm{K}<0.5$. the bearing capacity decreases with the increase of groove width ratio, with the increase of rotational speed, the hydrodynamic pressure produced between the axial and the axial force increases. When the rotating speed is zero, the surface pressure keeps $0.4 \mathrm{kN}$, mainly by the internal and external axial pressure and hydrodynamic pressure to provide bearing. Fig. 4b shows that with 
the increase of the depth of the groove, the opening force of the end face is reduced and the speed of the reduction is gradually reduced. When the groove depth $\mathrm{hg} \geq 3 \mu \mathrm{m}$, the sliding bearing remained essentially constant at $0.6 \mathrm{kN}$.

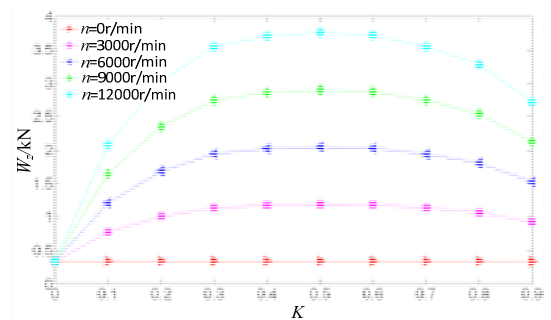

(a)Effect of groove width ratio on bearing capacity

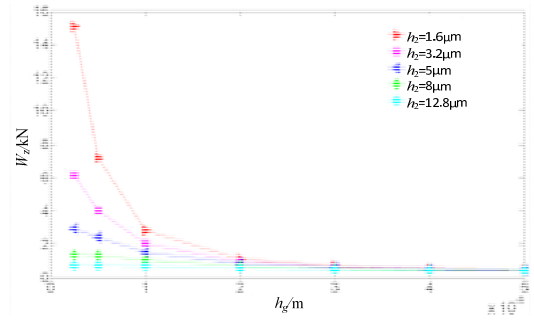

(b)Effect of groove depth on bearing capacity

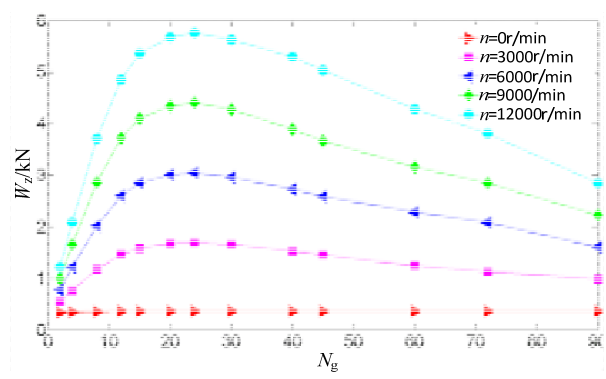

(c) Effect of groove number on bearing capacity

Figure 4. Effect of groove parameters on bearing capacity of axial bearing

The influence of the number of grooves on the bearing capacity shows in Fig. 4c. It can be seen that when the number of groove reaches 24 , the bearing capacity of the surface is maximum. When the number of grooves is less than 24, the bearing capacity increases with the increase of the number of grooves, and the number of grooves is greater than 24 , the bearing capacity decreases with the increase of the number of grooves.

\section{Friction Torque Analysis}

The effect of the groove depth on the friction torque of the axial bearing is shown in Fig. 5a. In the case of the other parameters unchanged, with the increase of the groove depth, the friction torque decreases and tends to flatten. When the lubrication gap is constant, the friction torque is proportional to the rotational speed and increases with the increase of rotational speed. When the speed is zero, the friction torque is zero.

The relationship between groove width and friction torque is shown in Fig. 5b. when the other parameters are constant, the friction torque increases with the increase of the slot width ratio, and the growth is slow when the groove width ratio is less than 0.5 , when the groove width is larger than 0.5 , the friction torque of the larger increase, which is due to the groove width ratio increases, the amount of leakage of lubricating oil increases, resulting in the decrease of bearing capacity of oil film, film thickness, thrust washers will gradually transition from full film lubrication to mixed lubrication; when the slot width ratio is constant, with the increase of speed, the friction torque increases, the speed is zero, and the friction torque is zero. 


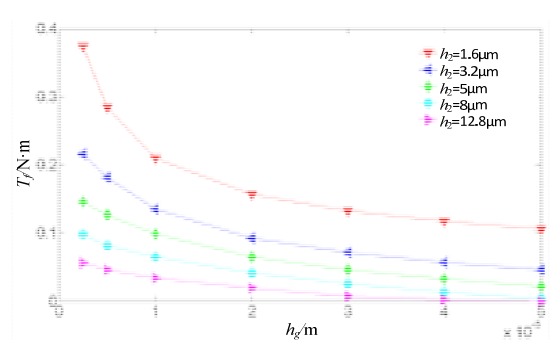

(a)Effect of groove depth on friction torque

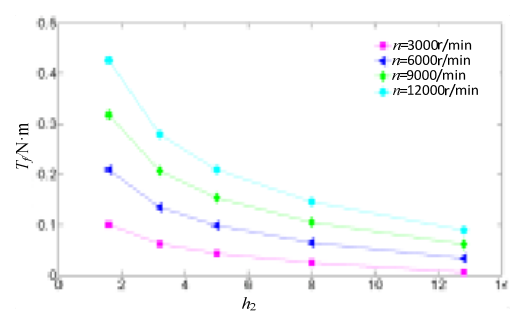

(c) Effect of lubrication gap on friction torque

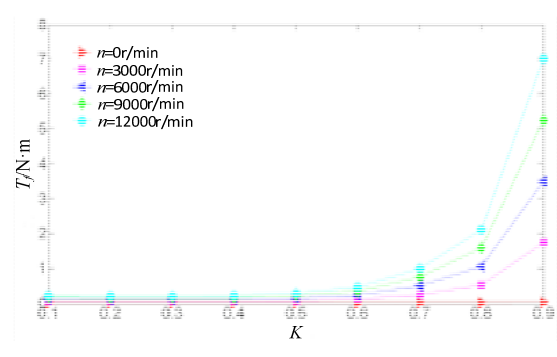

(b) Effect of groove width ratio on friction torque

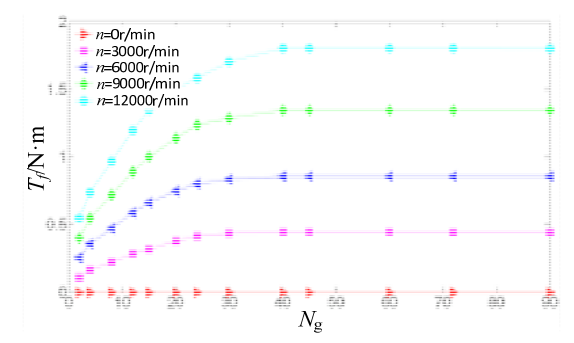

(d)Effect of groove number on friction torque

Figure 5. Effect of groove parameters on friction torque of axial bearing surface

Fig. $5 \mathrm{c}$ shows that with the increase of the oil film clearance, the thickness of the film between the axial bearing increases (in the whole oil film lubrication), the friction torque continues to decrease, and gradually tends to smooth. Fig. 5d illustrates the relationship between the number of slots and the torque. With other parameters under constant conditions, when the number of grooves is less than 40, the friction torque increases with the increase of the number of grooves. When the number of grooves is greater than or equal to 40 , the friction torque is basically unchanged (or decreased gradually, and the amount of reduction is very small).

\section{Lubricant Flow Analysis}

Fig. $6 \mathrm{a}$ is the effect of the groove width ratio on the lubricating oil flow. When the lubrication gap and other parameters are unchanged, the change of the groove width ratio has little effect on rate of oil flow. When the groove width $\mathrm{K}<0.5$, with the value increase of $\mathrm{K}$, surface flow decreased slightly, and the groove width ratio is $K>0.5$, the surface flow increases with the increase of $K$. But the increment is very small, the groove width is equal to the ratio of $\mathrm{K}$ reached the minimum rate of oil flow amount of 0.5 , mainly due to the same groove number and groove depth, the amount of flow of fluid mainly depends on the size of the gap in increasing axial bearing clearance, lubrication flow rate increases with the increase range rapidly. Fig. $6 \mathrm{~b}$ shows the relationship between groove depth and flow. Lubricant flow increases rapidly with increasing groove depth, the lubrication gap increases, the rapid increase in oil flow.

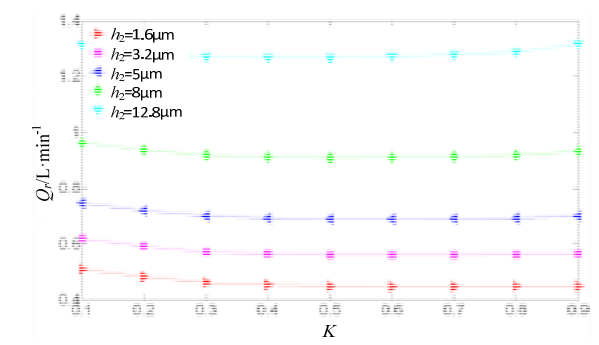

(a)Effect of groove width ratio on rate of oil flow

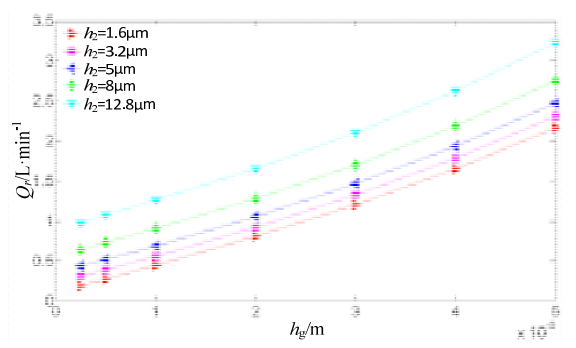

(b)Effect of groove depth on rate of oil flow 


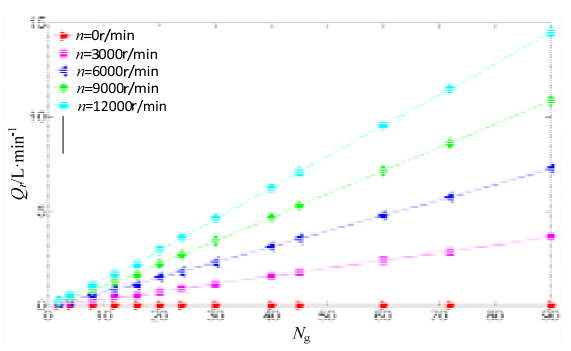

(c)Effect of groove number on rate of oil flow

Figure 6. Effect of groove parameters on rate of oil flow

Fig. 6c Shows the flow and the relationship between rotation speed and groove number. With the increase of slot number, lubricating rate of oil flow increasing, however, when the groove depth is more than $30 \mu \mathrm{m}$, the rate of increase of flow larger. The higher speed brings increased oil flow, however, when speed is zero, the leakage is greater than zero, that is due to the existence of axial pressure difference between internal and external.

\section{Conclusions}

The fluid pressure generated by the axial bearing can satisfy the axial bearing force requirements of planetary gears, and axial bearing fluid inlet and outlet pressure difference can produce greater bearing capacity. The influence of groove parameters on the bearing capacity was analyzed. It was considered that the groove-width ratio is 0.5 and the groove-number is 24 , which bring the maximum bearing capacity.

The higher the rotational speed of the planetary gear, the greater the friction torque and the greater the lubricating oil flow. The increase of axial clearance and groove depth or the reduction of groove width ratio and the number of groove can reduce the friction between the working surface of the planetary gear and axial bearing. Increasing the groove depth, lubrication gap, and number of groove can increase fluid flow.

In order to reduce the loss caused by the friction of the recommended groove depth value of $10 \sim$ $40 \mu \mathrm{m}$, considering previous work in bearing of planetary gear to wear stage, the actual processing of groove depth than the larger. When the groove depth is greater than $30 \mu \mathrm{m}$ to make the oil flow increases linearly, resulting in reduced bearing capacity of the axial bearing. Groove number and groove depth on the fluid flow greatly affected in the actual processing should take a small value. Increasing the lubrication clearance of the oil film bearing capacity decreases rapidly, mainly due to the sudden increase of the fluid flow. Considering the oil film gap about $5 \mu \mathrm{m}$ can reach the bearing capacity and friction torque, the end of the best balance of discharge capacity.

\section{Acknowledgement}

This research was financially supported by the National Foundation of Hebei Province, China(E2014402047) .

\section{References}

[1] Hong-wei WANG, Biao MA, Yan-he LI. A Study on the Mixed Lubrication Characteristics of Journal Bearing for High-speed Planet Gears in Tracked Vehicles [J]. Automotive Engineering,2014,03:328-333.

[2] Guan-hui HU, Ya-li CHOU. Principle and Maintenance of Automatic Transmission. Machinery Industry Press, 2006. 
[3] Salama. M. E., 1950, "The Effect of Macro-roughness on the Performance of Parallel Thrust Bearing," Proc. Inst. Mesh. Eng., 163, P. 149.

[4] Ulezelski J C, Evans D G, Haka R J et al. Needle bearing axial thrust study [J]. SAE Technical Paper Series, 830568, 1983.

[5] Jackson R L, Green I. The Behavior of thrust washer bearings considering mixed lubrication and asperity contact[J]. Tribology Transactions, 2006, 49(2): 233-247.

[6] To HimYu, Farshid Sadeghi. Groove Effect on Thrust Washer Lubrication[J]. Journal of Tribology,2001, Vol.123:295-304.

[7] Ju-tao ZHOU, Xiao-jun ZHOU, Fu-chun YANG. Study on Axial Force of Planetary Gear within Compound Planetary Spur Gear Set [J]. Mechanical Engineer, 2010(02):21-24.

[8] Fu-chun YANG. Study on dynamic characteristics of compound planetary gear and wear mechanism of its thrust washer [D].Zhejiang University, 2009.

[9] Song Pengyun, Chen Kuangmin, Dong Zongyu, Wu Zhiyu. Numerical Anaalysis of the Pressure on the Face of a Radial Groove Mchanical for Liquid[J]. Journal of Sichuan Union University(Engineering Scirnce Edition),1999,02:122-128.

[10] Ding-hua LIU, Ji-bin HU. Effect of cavitation model on the performance of radial grooved face seals [J]. Transactions of Beijing Institute of Technology. 2012,(11):1101-1104.

[11] Ji-bin HU, Ding-hua LIU, Chao WEIi. Numerical Simulation for Cavitation of Radial Grooved Face Seals [J]. Tribology, 2011,31(6):551-556.

[12] Hong-wei WANG, Biao MA, Xi-jing ZHAO, et al. Hydrodynamic lubrication characteristics of planet gear thrust washer used in high- speed planetary transmission [J]. Journal of Drainage and Irrigation Machinery Engineering. 2012,30(1):69-74.

[13] Bo-xian CHEN, Zu-gan QIU, Hui-sheng ZHANG. Fluid Llubrication Theory and its Application [M]. Beijing: Mechanical Industry Press, 1991. 